\title{
Efecto del uso de una somatotropina bovina recombinante (STbr) en vacas lecheras a pastoreo bajo condiciones tropicales ${ }^{\#}$
}

\author{
Effect of the use of a recombinant bovine somatotropin (rbST) in dairy grazing cows in a tropical \\ environment
}

\author{
A Vargas ${ }^{1}$, CA Osorio $^{1}$, J Loaiza $^{1}$, NA Villa ${ }^{2}$, A Ceballos ${ }^{2 *}$ \\ ${ }^{1}$ Departamento de Sistemas de Producción, Universidad de Caldas, AA 275, Manizales, Colombia. \\ ${ }^{2}$ Grupo de Investigación Salud Productiva en Bovinos, Porcinos y Equinos, Universidad de Caldas, AA 275, Manizales, Colombia.
}

\section{SUMMARY}

This study deals with the evaluation of the effect of a recombinant bovine somatotropin (rbST) on dairy production and the metabolic status of dairy cows. Twenty crossbreed (Harton del Valle x Holstein) lactating cows (12 weeks of lactation) were selected at random, and separated into two groups ( $\mathrm{n}=10$ each), control group (GC) and treatment group (GT). The GT received $500 \mathrm{mg}$ of rbST twice per month until 24 weeks of lactation. Daily milk yield was recorded every day until the end of the study, body condition score was evaluated, and the blood serum concentrations of glucose, cholesterol, and urea were analyzed, and milk urea was determined. Comparisons among groups to evaluate the effect of treatment and sampling period were made by using an ANOVA test. Milk production was higher $(7.4 \%)$ in the GT $(\mathrm{P}<0.05)$. Body condition score was similar between groups $(\mathrm{P}>0.05)$. Mean blood serum concentration of glucose and cholesterol was similar between groups $(\mathrm{P}>0.05)$. There was a tendency for the concentration of urea in the milk $(\mathrm{P}=0.20)$ to be lower in the GT. The use of rbST induced a higher milk yield in dairy cows in a tropical environment, but the nutritional requirements of the cows in both groups were higher and the feed intakes were not sufficiently high to support these additional requirements.

Palabras clave: bovinos, somatotropina recombinante, leche, metabolitos.

Key words: bovine, recombinant somatotropin, milk, metabolites.

\section{INTRODUCCION}

La hormona del crecimiento o somatotropina (ST) es una hormona proteica producida en las células acidófilas de la pituitaria anterior (Bauman 1992, Etherton y Bauman 1998, Prado y col 2003), cuya producción está regulada por las hormonas hipotalámicas, factor liberador de somatotropina y la somatostatina (Etherton y Bauman 1998, Prado y col 2003).

Hacia el inicio de la década de los años 80 se sintetizó somatotropina bovina por medio de tecnología con DNA recombinante (STbr), clonando un segmento específico de DNA bovino en la bacteria Escherichia coli $\mathrm{K}-12$, donde la molécula resultante mostró ser biológicamente idéntica a la natural (Bauman 1992, Chalupa y col 1996). La utilización de la STbr ha demostra-

Aceptado: 16.08.2005

\# Financiado por la Vicerrectoría de Investigaciones y Postgrados de la Universidad de Caldas y Schering-Plough SA, Bogotá DC.

* Grupo de Investigación Salud Productiva en Bovinos, Porcinos y Equinos, Universidad de Caldas, AA 275, Manizales, Colombia. Correo electrónico: aleceballos@ucaldas.edu.co do tener un efecto similar al que presenta la hormona natural sobre la producción de leche (Bauman 1992). En la glándula mamaria se produce un aumento de la síntesis láctea debido a una mayor captación de los nutrientes utilizados para su secreción y a un aumento en la actividad secretora de las células y la perfusión sanguínea (Bauman 1992, Bauman y Vernon 1993, Prado y col 2003). No obstante, la magnitud del incremento en la producción como respuesta a la administración de STbr está influenciada por factores internos y externos, como son la temperatura ambiente, el manejo general del rebaño, el período de lactancia, el potencial genético y la cantidad de leche producida (Bauman 1992, Prado y col 2003).

Otros efectos metabólicos que se han observado al utilizar STbr son el aumento de la gluconeogénesis hepática y la disminución en la actividad de la insulina para inhibirla (Prado y col 2003); la glucosa adicional es usada por la glándula mamaria como precursor de lactosa explicando el aumento en producción atribuido a la hormona. En el tejido adiposo disminuye la lipogénesis basal si se está en balance energético positivo e incrementa la lipólisis basal en balance energético negativo (Bauman 1992). Todo lo anterior conduce a una elevación de la 
energía disponible para la producción de leche, mejorando además la eficiencia alimenticia para la producción (Bauman 1992, Bauman y Vernon 1993, Lanna y col 1995). Pese a lo anterior, no se han observado alteraciones de la composición proteica de la leche (Bauman y Vernon 1993).

Se ha señalado que la producción de leche puede incrementarse hasta un 33\% (Lanna y col 1995), habiéndose reportado valores más bajos que pueden fluctuar entre $10 \%$ (Etherton y Bauman 1998) y 25,3\% (Thomas y col 1991), efecto que ha sido explicado por los fenómenos metabólicos descritos anteriormente. El incremento es gradual durante los primeros días, alcanzando la máxima producción una semana después; pero si el tratamiento se termina, la producción retorna a valores similares a los previos al tratamiento en un período de tiempo equivalente al que duró el uso de la hormona (Bauman y Vernon 1993).

Otros efectos metabólicos no asociados con la producción o la calidad de la leche han sido la reducción en la presentación de enfermedades metabólicas como hígado graso o cetosis (Bauman y Vernon 1993). Pese a ello, se ha descrito que el efecto de la STbr es insuficiente para lograr la prevención de casos de fiebre de leche pese a la inducción de una mayor movilización de calcio (Eppard y col 1996). Otros estudios han señalado que el uso de STbr indujo un mayor riesgo relativo para la presentación de problemas podales y necesidad de terapia antibiótica en casos de mastitis en vacas tratadas con 41,2 mg de STbr por día (Chalupa y col 1996).

En consideración a lo anterior, el objetivo de este estudio fue determinar el efecto de la utilización de una fuente comercial de somatotropina bovina recombinante (STbr) en vacas a partir de la semana 12 de lactancia y mantenidas en condiciones tropicales, sobre la producción de leche, condición corporal, concentración sérica de glucosa, colesterol y urea en suero y leche.

\section{MATERIAL Y METODOS}

Ubicación. Para la realización de este estudio se seleccionó una explotación lechera en la zona rural de Candelaria (Valle del Cauca), Colombia ( $3^{\circ} 27^{\prime}$ LN y $76^{\circ} 25^{\prime}$ LO). Zona clasificada según las zonas de vida de Holdridge como Bosque Seco Tropical, ubicada a 1.000 msnm, con temperatura media de $27^{\circ} \mathrm{C}$, una pluviosidad inferior a $1.000 \mathrm{~mm} /$ año y con un $70 \%$ de humedad relativa.

El sistema productivo del rebaño seleccionado se clasificó como lechería especializada en pastoreo intensivo sobre una superficie de 146 hectáreas (6 animales/ha) más suplementación, donde el ordeño se realizaba sin apoyo del ternero. Predominaba el cruzamiento entre la raza criolla colombiana Hartón del Valle y Holstein, con un promedio de producción de $11 \mathrm{~L} /$ vaca en dos ordeños diarios.
La alimentación estaba basada en pastoreo de Estrella (Cynodon plechtostachyus), con un período de recuperación de 30 días previa fertilización según el análisis de suelos, con una mezcla de nitrógeno, fósforo y potasio. El sistema de uso de la pradera era pastoreo rotacional en franjas. Las vacas se suplementaban en el momento del ordeño con alimentos concentrados importados al predio (proteína cruda entre $16 \%$ y $18 \%$, energía metabolizable: $2,8 \mathrm{Mcal} / \mathrm{kg} \mathrm{MS}$, fibra cruda: máximo $8 \%$ ), ofreciendo un $\mathrm{kg}$ de concentrado por tres litros de leche siempre que la producción fuera superior a nueve litros, ofreciendo máximo ocho $\mathrm{kg}$ de concentrado por día. Además, se suplementaba con una mezcla de minerales a razón de $120 \mathrm{~g} / \mathrm{vaca} /$ día en el momento del ordeño, en los potreros se disponía de sal común $(\mathrm{NaCl})$ y agua para consumo a voluntad.

Tratamientos. Se seleccionaron 20 vacas según su producción de leche, estado de lactancia (semana 12) y número de partos. En un diseño de bloques completos al azar, las vacas se asignaron en uno de dos grupos: control $(\mathrm{GC}, \mathrm{n}=10)$ que no recibió ningún tratamiento y tratado (GT, $n=10)$, que recibió cada 14 días una inyección subcutánea $(2 \mathrm{~mL})$ de un preparado comercial de somatotropina bovina recombinante (STbr) (Bomate-S ${ }^{\circledR}$, LG Chem Investment Ltd., Corea del Sur para ScheringPlough SA, Bogotá DC), compuesta por $500 \mathrm{mg}$ de STbr, 1665 UI de vitamina E y $166,5 \mathrm{mg}$ de lecitina. El tratamiento se terminó en la semana 24 de lactancia.

La producción de leche se pesó diaria e individualmente en las vacas de ambos grupos antes de la primera aplicación de la hormona continuando hasta finalizar el estudio, posteriormente se obtuvo un promedio semanal. También, cada mes se calificó la condición corporal hasta completar cuatro evaluaciones, usándose una escala de 1 a 5 (NRC 2001), donde la calificación 1 corresponde a una vaca delgada y 5 a un animal obeso. La condición corporal promedio al inicio del ensayo fue $2,9 \pm 0,1$.

Muestras. Antes de la primera aplicación del producto, y posteriormente, cada mes, durante cuatro meses, se extrajeron $5 \mathrm{~mL}$ de sangre por animal mediante venopunción coccígea, usando tubos al vacío sin anticoagulante (Vacutainer ${ }^{\circledR}$, Becton-Dickinson, Franklin Lakes, USA); las muestras se refrigeraron y enviaron dentro de 12 horas al Laboratorio de Patología Clínica Veterinaria de la Universidad de Caldas. Las muestras se centrifugaron a $3.000 \mathrm{rpm}$ por 15 minutos para separar el suero, envasándolo y conservándolo $\mathrm{a}-20^{\circ} \mathrm{C}$ hasta su análisis. Igualmente, se tomaron $100 \mathrm{~mL}$ de leche de cada vaca, las muestras se mezclaron y homogeneizaron, enviando 250 $\mathrm{mL}$ de cada grupo en las mismas condiciones descritas y al mismo laboratorio. La muestra se centrifugó doblemente a $3.000 \mathrm{rpm}$ por 20 minutos para separar la grasa, posteriormente se determinó la concentración de urea en leche para el grupo. 
Análisis. Se determinaron por colorimetría las concentraciones de glucosa (GOD-PAP), colesterol (CHODPAP) y urea (Ureasa Berthelot modificado), para lo cual se emplearon reactivos comerciales (BioSystems ${ }^{\circledR}$, Barcelona, España). Las lecturas de las muestras se realizaron en un espectrofotómetro BioSystems BTS 330 (BioSystems $^{\circledR}$, Barcelona, España). Los resultados se expresan según el Sistema Internacional de Unidades en $\mathrm{mmol} / \mathrm{L}$

Análisis estadístico. El análisis se efectuó usando el PROC MIXED de SAS (SAS Inst. Inc. Cary, NC, USA) empleando también la opción REPEATED para incluir la matriz de varianzas-covarianzas entre medidas repetidas en un mismo individuo. El diseño del estudio correspondió a un estudio completamente al azar, donde se determinaron el promedio ( $\overline{\mathrm{X}})$, error estándar $(\mathrm{EE})$, intervalo de confianza (IC) al $95 \%$ y el coeficiente de variación $(\mathrm{CV})$. Se empleó un análisis de varianza para determinar el efecto del tratamiento, el tiempo posterior a la aplicación y su interacción sobre las diferentes variables estudiadas, según el modelo:

$$
X_{i j}=\mu+A_{i}+B_{j}+(A x B)_{i j}+\varepsilon_{i j}
$$

Donde $\mathrm{X}_{\mathrm{ij}}$ : observación, $\mu$ : media general, $\mathrm{A}_{\mathrm{i}}$ : tratamiento, $\mathrm{B}_{\mathrm{j}}$ : momento del muestreo (período), $(\mathrm{AxB})_{\mathrm{ij}}$ : interacción entre tratamiento y momento del muestreo (período), y $\varepsilon_{\mathrm{ij}}$ : error, fijándose $\mathrm{P}<0,05$ como nivel de significancia.

\section{RESULTADOS}

Producción y condición corporal. La producción promedio de leche por día fue diferente entre los grupos $(\mathrm{P}=0,002)$, encontrándose que la cantidad diaria a lo largo del estudio fue mayor en el GT (cuadro 1). El aumento observado en la producción promedio para el GT fue $7,4 \%$; después de la aplicación de la hormona la producción aumentó una semana después de iniciado el trata-

Cuadro 1. Promedio, error estándar, intervalo de confianza y coeficiente de variación para la producción de leche en vacas no tratadas (GC) y tratadas con STbr (GT) durante 12 semanas de lactancia.

Mean, standard error, confidence interval, and variation for milk yield (kg/day) in dairy cow controls (GC) and those treated (GT) with rbST during 12 weeks of lactation.

\begin{tabular}{cccc}
\hline & \multicolumn{3}{c}{ Producción láctea $(\mathrm{kg} /$ día) } \\
\cline { 2 - 4 } & GC & GT & $\mathrm{P}^{*}$ \\
\hline$\pm \mathrm{EE}$ & $14,9 \pm 0,4$ & $16,0 \pm 0,3$ & 0,002 \\
$\mathrm{IC}(95 \%)$ & $14,1-15,8$ & $15,3-16,8$ & \\
CV $(\%)$ & 9,0 & 7,3 & \\
\hline
\end{tabular}

* Diferencias entre tratamientos. miento, observándose el mismo patrón después de cada aplicación (figura 1).

La condición corporal fue similar entre los grupos, observándose a lo largo del estudio un promedio en la condición de $2,7 \pm 0,1$ y $2,8 \pm 0,1$ para los grupos tratado y control respectivamente $(\mathrm{P}=0,122)$. Se encontró una reducción de la condición en ambos grupos a lo largo del estudio, obteniéndose la calificación más baja al finalizar el estudio en la semana 24 de lactancia $(\mathrm{P}=0,001)$.

Metabolitos sanguíneos. La concentraciones séricas de glucosa y colesterol no presentaron diferencias entre los grupos ( $\mathrm{P}>0,05)$ estudiados (cuadro 2). Con respecto a la concentración de urea en suero no se presentaron diferencias significativas $(\mathrm{P}>0,05)$ entre los grupos, mientras que en la leche hubo una tendencia $(\mathrm{P}=0,200)$ a observar valores inferiores en el GT (cuadro 2). Igualmente, no hubo variaciones para la concentración de glucosa y urea en suero y leche según el momento del muestreo $(\mathrm{P}>0,05)$.

Por el contrario, la concentración de colesterol aumentó en forma sostenida en ambos grupos de vacas a partir del inicio del tratamiento, observándose la mayor concentración promedio para ambos grupos en el último período de muestreo $(\mathrm{P}=0,030)$ (figura 2 ).

\section{DISCUSION}

La utilización de STbr produjo un aumento de 7,4\% en la producción de leche diaria promedio del GT con respecto al GC (cuadro 1), lo que ha sido observado en otros trabajos donde se han obtenido incrementos similares; así, en Brasil el uso de STbr en dosis entre 250 y $500 \mathrm{mg} /$ vaca con la misma frecuencia de uso descrita en este trabajo, causó un aumento en la producción entre $3,0 \%$ y $6,4 \%$ en la tercera semana post-tratamiento, lográndose la máxima producción en la sexta semana

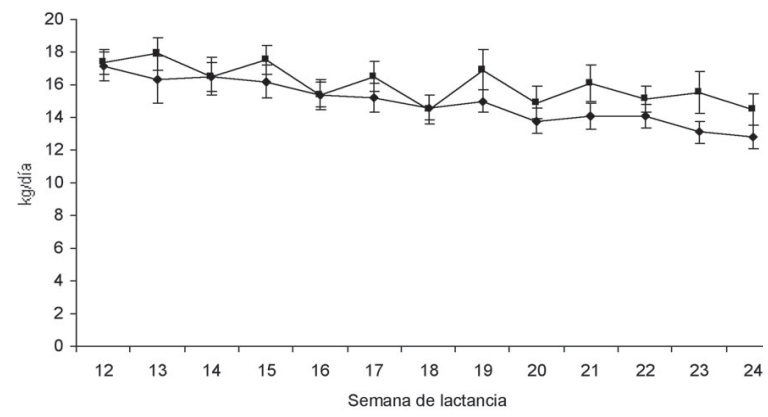

Figura 1. Promedio ( $\overline{\mathrm{X}} \pm \mathrm{EE}$ ) para la producción de leche por día ( $\mathrm{kg} /$ día) entre la semana 12 y 24 de lactancia en vacas no tratadas (GC ) y tratadas con STbr (GTa).

Mean $(\bar{X} \pm \mathrm{SE})$ milk yield per day $(\mathrm{kg} /$ day) between 12 and 24 weeks of lactation in dairy cow controls (GC $\bullet$ and those treated (GTच) with rbST. 
Cuadro 2. Promedio, error estándar e intervalo de confianza (IC) para la concentración sérica de glucosa, colesterol y urea (suero y leche) en vacas no tratadas (GC) y tratadas con STbr durante 12 semanas de lactancia.

Mean, standard error, and confidence interval for glucose and cholesterol in blood serum, and urea (blood serum and milk) in lactating dairy cow controls (GC) and those treated (GT) with rbST during 12 weeks of lactation.

\begin{tabular}{lccc}
\hline Metabolito & $\mathrm{GC}$ & $\mathrm{GT}$ & $\mathrm{P} *$ \\
\hline $\begin{array}{l}\text { Glucosa }(\mathrm{mmol} / \mathrm{L}): \\
\quad \pm \mathrm{EE}\end{array}$ & $2,9 \pm 0,1$ & $2,8 \pm 0,1$ & 0,644 \\
$\mathrm{IC}(95 \%)$ & $2,7-3,0$ & $2,6-3,0$ & \\
& & & \\
Colesterol (mmol/L): & & & \\
$\quad \pm \mathrm{EE}$ & $3,6 \pm 0,2$ & $3,7 \pm 0,2$ & 0,930 \\
IC (95\%) & $3,2-4,1$ & $3,2-4,1$ & \\
$\quad$ & & & \\
Urea $(\mathrm{mmol} / \mathrm{L}):$ & & & \\
$\quad \pm \mathrm{EE}$ & $4,1 \pm 0,2$ & $3,9 \pm 0,2$ & 0,378 \\
IC (95\%) & $3,7-4,7$ & $3,6-4,2$ & \\
$\quad$ & & \\
Urea en leche (mmol/L): & & & \\
$\quad \pm \mathrm{EE}$ & $6,7 \pm 0,6$ & $5,5 \pm 0,6$ & 0,200 \\
IC (95\%) & $4,3-8,6$ & $3,6-7,4$ & \\
\hline
\end{tabular}

* Diferencias entre tratamientos.

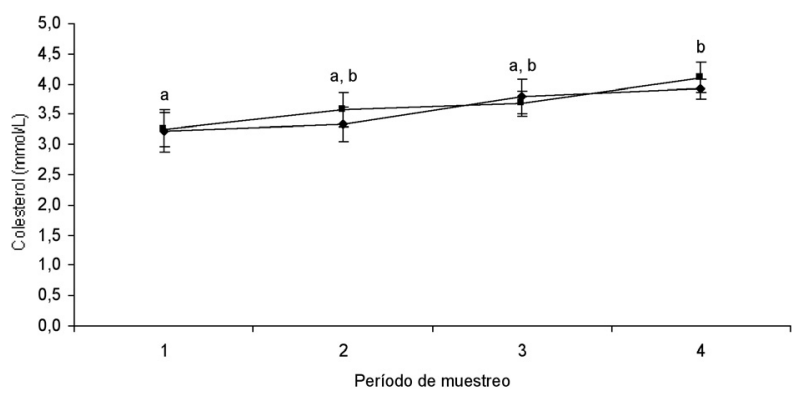

Figura 2. Variación mensual de la concentración sérica de colesterol $(\overline{\mathrm{X}} \pm \mathrm{EE})$ en vacas no tratadas $(\mathrm{GC} \bullet)$ y tratadas con STbr (GT匹) durante 12 semanas de lactancia. ${ }^{\text {a,b}}$ Significan diferencias entre períodos $(\mathrm{P}<0,05)$.

Variation of cholesterol ( $\overline{\mathrm{X}} \pm \mathrm{EE}$ ) in blood serum of dairy cow controls (GC ) and those treated (GT $\mathbf{}$ ) with rbST during 12 weeks of lactation. ${ }^{\mathrm{a}, \mathrm{b}}$ Means different among periods $(\mathrm{P}<0,05)$.

(Dos Santos y col 2001), obteniéndose la mejor respuesta con $350 \mathrm{mg}$ de STbr/vaca. Pese a ello, hay otros estudios donde se han observado aumentos de producción superiores a los descritos, alcanzando entre $10 \%$ y $25 \%$ más respecto a los grupos control (Peel y Bauman 1987, Lotan y col 1993, Etherton y Bauman 1998).

En este estudio no se observaron incrementos mayores posiblemente por factores relacionados con el consumo de materia seca y la satisfacción de los requerimientos nutricionales que impone el aumento de la producción, ya que el uso de STbr eleva las necesidades nutricionales (Bauman y Vernon 1993) y se requiere un incremento en el consumo (Chalupa y col 1996). Además, debe considerarse que las gramíneas tropicales frente a forrajes propios de países templados no ofrecen la calidad nutricional suficiente para satisfacer las necesidades de vacas en pastoreo, especialmente en lo referente a energía metabolizable, debiendo incrementarse la suplementación con alimentos concentrados u otros suplementos, cosa que no ocurrió en este estudio, ya que la relación leche:concentrado se mantuvo independiente del incremento en la producción observado en el GT.

En la condición corporal se observó una disminución en ambos grupos a lo largo del estudio, no observándose diferencias según el tratamiento. El aumento de los requerimientos nutricionales impuesto por la producción de leche, especialmente energéticos, induce a la vaca a recurrir a sus propias reservas para mantener la producción cuando no se cubren en forma adecuada, perdiéndose así la condición corporal (NRC 2001). A diferencia de lo anterior, en otros estudios se ha descrito que al transcurrir más días post-tratamiento y con dosis crecientes de STbr, la vaca pierde peso, pero son cambios que según los autores no necesariamente reflejan la depleción o repleción de reservas corporales (Nytes y col 1990, Chalupa y col 1996). Estos mismos autores señalan que en otras investigaciones se ha descrito que la disminución de la condición corporal está relacionada con la estimulación que ejerce la STbr sobre la partición de las calorías destinadas a la producción de leche a expensas de las disponibles para depositar grasa. Para corroborar lo anterior, se ha descrito que las vacas tratadas con STbr pierden más condición corporal que las vacas no tratadas (Remond y col 1991, Thomas y col 1991, Stehr y col 2001). Pese a lo descrito, los valores de condición corporal observados fueron considerados como adecuados para el estado productivo en el cual se encontraban las vacas (Stehr y col 2001).

El uso de la STbr no produjo cambios significativos en la concentración sérica de glucosa, colesterol y urea (cuadro 2). En otros estudios igualmente se han encontrado concentraciones similares de glucosa entre los grupos tratados y no tratados con STbr (Prado y col 2003). Contrario a lo anterior, en otros experimentos se han atribuido cambios en la glicemia por la utilización de la hormona como reflejo de un incremento de la gluconeogénesis para generar la energía extra y mantener el incremento en producción de leche (Bauman 1992, Etherton y Bauman 1998).

La ausencia de un cambio significativo en la glicemia en los resultados obtenidos pudo deberse a una falla en los mecanismos gluconeogénicos de las vacas del estudio o a una mayor utilización de la glucosa por parte de la glándula mamaria para la síntesis láctea o a una disminución en el aporte de los sustratos necesarios en la ración para la síntesis de glucosa. El hígado presenta una reserva limitada de glucógeno para mantener la demanda de glucosa en la lactancia, lo que unido a un incre- 
mento en la demanda, por efecto del uso de la STbr, haría que la glicemia no aumente por una escasez en la reserva de sustratos (Etherton y Bauman 1998).

En cuanto al colesterol, se debe tener en cuenta que su concentración cambia según el balance energético, el estado productivo de la vaca (Bauman 1992) y puede observarse una variación circadiana debida a factores como alimentación, producción y síntesis (Bitman y col 1990). En este estudio no se observaron cambios según el tratamiento (cuadro 2), manteniéndose en valores superiores a los descritos para vacas lactantes, los que deben ubicarse por encima de 2,6 $\mathrm{mmol} / \mathrm{L}$ para el preparto y postparto respectivamente (Van Saun 1997). En otros estudios donde se ha evaluado el efecto de hormona en ganado productor de carne se ha observado que la concentración sérica de colesterol así como su contenido en músculo y grasa de depósito no han presentado cambios entre grupos tratados y no tratados (Holzer y col 2000, Prado y col 2003).

Variables como la composición de la dieta, el tiempo posterior a la ingesta del alimento, la edad, la raza, la fase de gestación y lactancia pueden haber determinado la falta de cambio en la concentración de colesterol entre los grupos; además, cuando los animales tratados se encuentran en balance energético positivo al inicio del tratamiento con STbr, el efecto sobre la lipogénesis es inhibitorio, redistribuyendo las grasas para la síntesis de leche (Bauman y Vernon 1993). Las vacas que se consideraron en este trabajo se encontraban en una fase de lactancia posterior al pico de producción cuando se administró la hormona, es decir, se encontraban en balance energético (figura 2), lo que podría haber influido en la respuesta lipídica al tratamiento.

La ausencia de efecto del tratamiento sobre los metabolitos glucosa y colesterol puede también estar relacionada con el momento de la toma de la muestra, ya que se ha señalado que el efecto hormonal después de la última inyección empieza a declinar tan rápido como 24 horas después (Lee y col 2000). En este trabajo las muestras se tomaron mensualmente, donde habían pasado mínimo dos semanas después de la inyección de la hormona al momento de la muestra.

La concentración de urea en sangre y leche encontrada en ambos grupos fue similar (cuadro 2), pese a ello se observó una tendencia a presentar un mayor valor de urea en leche en el GC, no superándose el límite superior referencial (Wittwer y col 1999). Se ha indicado que el balance nitrogenado y su reflejo en la concentración de urea en leche cuando se administra STbr depende de factores inherentes a la composición de la dieta, como cantidad de proteína degradable y su relación con la energía fermentable, entre otros (Bauman 1992). Lo anterior llevaría a señalar que frente a la tendencia a observar valores de urea en sangre y leche mayores en el GC, el consumo de proteína, el balance energético o la relación entre ambos era inadecuado para este grupo. También puede señalarse que en el GT se haya presentado una mejor utilización del nitrógeno de la dieta (Oldenbroek y col 1993), lo que requiere estudios posteriores para su comprobación, donde se controlen variables como contenido de proteína y energía fermentable.

Bajo estas condiciones, es posible señalar que la utilización de STbr a partir de la semana 12 de lactancia y hasta la semana 24 , en vacas con baja producción y mantenidas en pastoreo bajo condiciones tropicales, tuvo un efecto positivo sobre la cantidad de leche producida, sin alcanzar los incrementos descritos en la literatura, posiblemente por efecto de la dieta que fue insuficiente para satisfacer los requerimientos nutricionales. Además, no se observaron diferencias en los indicadores sanguíneos del metabolismo de las vacas debido al uso de la hormona, excepto por una tendencia a observar valores inferiores de urea en leche en el GT que reflejarían una mejor utilización orgánica del nitrógeno de la ración.

\section{RESUMEN}

Para evaluar el efecto del uso de la somatotropina bovina recombinante (STbr) sobre la producción e indicadores metabólicos en vacas bajo condiciones tropicales, se seleccionaron al azar 20 vacas cruzadas (Hartón del Valle $\mathrm{x}$ Holstein) con 12 semanas de lactancia y mantenidas en pastoreo. Fueron distribuidas en dos grupos ( $\mathrm{n}=10$ por grupo) $\mathrm{y}$ asignadas a uno de dos tratamientos en un diseño de bloques completos al azar: grupo control (GC) no recibió STbr y grupo tratado (GT) recibió, por vía subctánea, $500 \mathrm{mg}$ de STbr cada dos semanas durante 12 semanas. Se hicieron pesajes diarios de leche, se evaluó la condición corporal y se determinó la concentración de glucosa, colesterol y urea en sangre y leche. Las comparaciones entre grupos para evaluar el efecto del tratamiento y el tiempo se hicieron mediante un ANDEVA. La producción de leche aumentó un $7,4 \%$ en el GT $(\mathrm{P}<0.05)$, no hubo diferencias en la condición corporal entre los grupos a través del ensayo $(\mathrm{P}<0,05)$. No se observaron diferencias entre la concentración de glucosa, colesterol y urea entre los grupos $(\mathrm{P}>0,05)$, mientras que la concentración de urea en leche presentó una tendencia $(\mathrm{P}=0,20)$ a mostrar valores inferiores en el GT. La utilización de STbr indujo una mayor cantidad de leche producida en vacas a pastoreo en el trópico.

\section{REFERENCIAS}

Bauman DE. 1992. Bovine somatotropin: Review of an emerging animal technology. J Dairy Sci 75, 3432-3451.

Bauman DE, RG Vernon. 1993. Effects of exogenous bovine somatotropin on lactation. Annu Rev Nutr 13, 437-461.

Bitman J, DL Wood, AM Lefcoufil. 1990. Rhythms in cholesterol, cholesteryl esters, free fatty acids, and triglycerides in blood of lactating dairy cows, milk secretion and mastitis laboratory livestock and poultry. J Dairy Sci 73, 948-955.

Chalupa W, B Vecchiarelli, DT Galligan, JD Ferguson, LS Baird, RW Hemken, RJ Harmon, CG Soderholm, ED Otterby, RJ Annexstad, JG Linn, WP Hansen, RJ Ehle, DL Palmquist, RG Eggert. 1996. Responses of dairy cows 
supplemented with somatotropin during weeks 5 through 43 of lactation. J Dairy Sci 79, 800-812.

Dos Santos RA, JC Teixeira, LR Abreu, JA Muniz, F Desresz. 2001. Efeito de diferentes doses de somatotropina bovina (Rbst) na produção e composição do leite. Ciênc Agrotec Lavras 25, 1435-1445.

Eppard PJ, JJ Veenhuizen, WJ Cole, PG Comens-Keller, GF Hartnell, RL Hintz, L Munyakazi, PK Olsson, RH Sorbet, TC White, CA Baile, RJ Collier, JP Goff, RL Horst. 1996. Effect of bovine somatotropin administered to periparturient dairy cows on the incidence of metabolic disease. J Dairy Sci 79, 2170-2218.

Etherton TD. 2000. The biology of somatotropin in adipose tissue growth and nutrient partitioning. J Nutr 130, 26232625.

Etherton TD, DE Bauman. 1998. Biology of somatotropin in growth and lactation of domestic animals. Physiol Rev 78, 745-761.

Holzer Z, Y Aharoni, A Brosh, A Orlov, F Buonomo. 2000. The influence of recombinant bovine somatotropin on dietary energy level related growth of Holstein-Friesian bull calves. J Anim Sci 78, 621-628.

Lanna DPD, KL Houseknecht, DM Harris, DE Bauman. 1995. Effect of somatotropin treatment on lipogenesis, lipolisis, and related cellular mechanisms in adipose tissue of lactating cows. J Dairy Sci 78, 1703-1712.

Lee KC, MJ Azain, DB Hausman, TG Ramsay. 2000. Somatotropin and adipose tissue metabolism: Substrate and temporal effects. J Anim Sci 78, 1236-1246.

Lotan E, H Sturman, JI Weller, E Ezra. 1993. Effects of recombinant bovine somatotropin under conditions of high production and heat stress. J Dairy Sci 76, 1394-1402.

National Research Council (NRC). 2001. Nutrient requirements of dairy cattle. $7^{\text {th }}$ ed., National Academy Press, Washington DC, USA.
Nytes AJ, DK Combs, GE Shook, RD Shaver. 1990. Response to recombinant bovine somatotropin in dairy cows with different genetic merit for milk production. J. Dairy Sci. 73, 784-791.

Oldenbroek JK, GJ Garssen, LJ Jonker, JID Wilkinson. 1993. Effects of treatment of dairy cows with recombinant bovine somatotropin over three or four lactations. J Dairy Sci 76, 453-467.

Peel CJ, DE Bauman. 1987. Somatotropin and lactation. J Dairy Sci 70, 474-485.

Prado I, WG Nascimento, JA Negrao, LP Rigolon, S De Souza, ML Doi Sakuno, GL Pessini. 2003. Recombinant bovine somatotropin (rBST) on hematologic aspects and metabolites of heifers $(1 / 2$ Nellore $\mathrm{x} 1 / 2$ Red Angus) blood, in feedlot. $R$ Bras Zootec 32, 465-472.

Remond B, M Cisse, A Ollier, Y Chilliard. 1991. Slow release somatotropin in dairy heifers and cows fed two levels of energy concentrate: Performance and body condition. $J$ Dairy Sci 74, 1370-1381.

Stehr W, B Twele, L Rosales. 2001. Uso de somatotropina recombinante en vacas lecheras. Arch Zootec 50, 419-422.

Thomas JW, RA Erdman, DM Galton, RC Lamb, MJ Arambel, JD Olson, KS Madsen, WA Samuels, CJ Peel, GA Green. 1991. Responses by lactating cows in commercial dairy herds to recombinant bovine somatotropin. J Dairy Sci 74, 945-964.

Van Saun R. 1997. Nutritional profiles: a new approach for dairy herds. Bov Pract 31, 43-50.

Wittwer FG, P Gallardo, J Reyes, H Opitz. 1999. Bulk milk urea concentrations and their relationships cow fertility in grazing dairy herd in sother Chile. Prevent Vet Med 38, 159-166. 